\title{
Paidéia: Relatório de Gestão - 2011
}

\author{
Manoel Antônio dos Santos ${ }^{1}$ \\ Universidade de São Paulo, Ribeirão Preto-SP, Brasil
}

O presente relatório tem como objetivo descrever indicadores que permitem dar visibilidade à gestão editorial da revista Paidéia no ano de 2011. Com a publicação desse documento mantém-se a tradição de apresentar à comunidade científica um balanço do que a revista produziu no último ano, dando visibilidade ao modus operandi do processo de tramitação dos manuscritos no decurso desse período.

A disseminação da produção científica é lastreada em um rigoroso processo de aferição por pares. Ao sistematizar informações relativas ao processo editorial, é possível localizar a contribuição da Paidéia no cenário contemporâneo das publicações científicas na área das Ciências Humanas, especialmente da Psicologia e áreas afins.

Este relatório está subdividido nas seguintes seções: Política editorial e critérios de arbitragem, Processo editorial - 2011 e Considerações finais.

\section{Política Editorial e Critérios de Arbitragem}

A Paidéia é publicação quadrimestral do Programa de Pós-graduação em Psicologia, da Faculdade de Filosofia, Ciências e Letras de Ribeirão Preto da Universidade de São Paulo - FFCLRP-USP. Tem como escopo e foco editorial publicar estudos relacionados à Psicologia e áreas afins. Os textos publicados devem enquadrar-se nas categorias: relato de pesquisa, estudo teórico e revisão sistemática da literatura. Até 2011 eram admitidas as seguintes categorias de manuscritos: relato de pesquisa, estudo teórico, relato de experiência profissional, revisão sistemática da literatura, comunicação breve, nota técnica e resenha. Essas categorias estão refletidas nos resultados divulgados no presente relatório.

Em 2011, a Paidéia completou 20 anos de existência. Desde sua fundação, em agosto de 1991, foram publicados 50 fascículos, do volume 1 ao 21, e seu acervo encontra-se totalmente disponibilizado, em acesso livre e gratuito assegurado pela Coleção SciELO. Os fascículos são marcados pela diversidade e abrangência dos temas e delineamentos contidos em seus artigos. A linha editorial é generalista, em respeito à diversidade teórico-metodológica, epistemológica e temática que permeia a área da Psicologia.

\footnotetext{
${ }^{1}$ Endereço para correspondência:

Prof. Dr. Manoel Antônio dos Santos. Universidade de São Paulo. Faculdade de Filosofia, Ciências e Letras de Ribeirão Preto. Departamento de Psicologia e Educação. Av. Bandeirantes, 3900. CEP: 14040-901. Ribeirão Preto-SP, Brasil. E-mail: masantos@ffclrp.usp.br
}

Em termos da classificação no sistema Qualis Periódico, na área da Psicologia (Comissão Qualis Periódico da Psicologia, 2012), a Paidéia ascendeu recentemente do estrato B1, no qual se encontrava até 2011, para A1, que é a posição mais elevada do ranking da avaliação, o que evidencia o reconhecimento do lugar destacado que esse periódico vem ocupando no conjunto das publicações científicas brasileiras. Esse nível de excelência também ficou patenteado por um outro indicador importante - no início de 2012 a revista foi uma das duas publicações nacionais contempladas com o Programa de Apoio da CAPES para alavancar a internacionalização dos periódicos científicos na área da Psicologia. Essa indicação foi patrocinada pela Comissão de Avaliação Qualis Periódicos e pela Coordenação de Área de Avaliação (Psicologia) da CAPES. O Qualis A1 e o fato de ter sido escolhida pela CAPES para fazer jus ao Programa de Apoio a periódicos com potencial para internacionalização são conquistas que consagraram a Paidéia como uma das principais revistas de Psicologia no panorama atual da Psicologia.

O prestígio angariado por este periódico junto à comunidade científica se deve à sua seletiva política de divulgação do conhecimento de qualidade. Esse trabalho de aferição rigorosa e criteriosa tem sido sustentado com o apoio de um Conselho Editorial altamente qualificado, estruturado em função das áreas e dos temas abordados nos artigos submetidos à apreciação. Esse Conselho é constituído por nomes expressivos da comunidade acadêmica, tanto no âmbito nacional como internacional. A tramitação dos manuscritos está a cargo de uma Comissão Editorial constituída por docentes vinculados ao Programa de Pós-graduação em Psicologia da FFCLRP-USP. Essa Comissão coordena um trabalho rigoroso de revisão de pares, lançando mão de consultores ad hoc com reconhecida competência, que têm a missão de avaliar e aprimorar os manuscritos submetidos pelos autores.

Além disso, para consolidar sua visibilidade no plano nacional e internacional, a Paidéia está indexada em 12 bases (ou portais de texto completo) nacionais e internacionais, a saber: SciELO - Scientific Eletronic Library Online (FAPESP/ BIREME), Scopus (Elsevier), PsycINFO - Psychological Abstracts (American Psychological Association), PASCAL L'Institut de l'Information Scientifique et Technique (INIST), LILACS - Literatura Latino-Americana e do Caribe em Ciências da Saúde (Centro Latino-americano y de Caribe de Informaciones em Ciências de la Salud), CLASE - Citas Latinoamericanas en Ciências Sociales y Humanidades (Universidad Nacional Autónoma de México), PSICODOC (Colégio Oficial de Psicólogos de Madrid / Universidad Complutense de Madrid), DOAJ - Directory of Open Access 
Journals, Ulrich's International Periodicals Directory, Catálogo Latindex, IRESIE (Universidad Nacional Autónoma de México) e Index-Psi Periódicos (CFP/PUCCAMP).

A submissão dos manuscritos é inteiramente informatizada e assegurada por meio do sistema eletrônico Submission, disponibilizado pela SciELO. A revista adota o sistema de avaliação blind review, que preserva a identidade dos autores e dos avaliadores. Os textos submetidos à apreciação devem enquadrar-se, prioritariamente, na categoria relato de pesquisa. Em cada fascículo são publicados cerca de 13 artigos originais de pesquisa. A partir de 2012 (novas submissões) não são mais admitidos relatos de experiência profissional, comunicação breve, nota técnica e resenha de livro. Isso porque a Comissão Editorial decidiu, como parte da nova política editorial da revista, investir maciçamente na captação de artigos que dêem visibilidade a resultados de pesquisas derivados de estudos empíricos originais, com delineamento quanti ou qualitativo. O potencial de gerar impacto na área de conhecimento também é um critério utilizado para seleção dos manuscritos para publicação.

No que concerne às fontes de financiamento, a Paidéia tem contado com aportes de recursos financeiros advindos regularmente do Programa de Pós-graduação em Psicologia da FFCLRP-USP, do Programa de Apoio às Publicações Científicas Periódicas da USP e do Programa de Apoio a Publicações Científicas do CNPq.

Ao final de cada fascículo são incluídas as normas de publicação, disponíveis em três idiomas: português, inglês e espanhol. Essas normas (Instruções aos Autores) também estão disponibilizadas na página da SciELO (http://www. scielo.br/paideia). O último fascículo de cada volume contempla a relação de assessores ad hoc, que durante o ano vigente contribuíram com a árdua tarefa de revisão dos manuscritos.

A versão impressa da Paidéia é distribuída gratuitamente para, aproximadamente, 160 bibliotecas de universidades públicas brasileiras vinculadas à Rede Brasileira de Bibliotecas da Área de Psicologia - ReBAP. Desse modo, os fascículos são disponibilizados para cursos de graduação em Psicologia, bem como de alguns cursos de Educação, além de países do exterior, como Portugal, Espanha, México e EUA. Isso significa que, por três vezes ao ano, são enviados exemplares para instituições de nível superior de grande parte dos estados brasileiros e algumas do exterior, bem como para Programas de Pós-graduação nas áreas de Psicologia ou Educação. Também são estabelecidas permutas com periódicos brasileiros e internacionais da área de Psicologia e Saúde. O último exemplar de cada volume também é encaminhado a todos os consultores ad hoc que colaboraram com a revista durante o ano, em reconhecimento à colaboração oferecida na emissão de pareceres. Nesse último fascículo (versão impressa) de cada volume disponibilizamos a relação dos consultores ad hoc que colaboraram com a revista durante o ano vigente. É um tributo que rendemos como forma de agradecimento aqueles que contribuíram com a revisão dos manuscritos submetidos à apreciação da revista.

No que concerne ao processo de arbitragem dos manuscritos, a Paidéia adota o mecanismo de revisão cega e dupla por pares de reconhecido saber e prestígio junto à comunidade científica. Desse modo, as identidades dos autores e dos assessores ad hoc são mantidas no mais absoluto sigilo. Os textos submetidos à avaliação devem estar de acordo com as orientações das normas de publicação e podem ser aceitos ou recusados pela Comissão Editorial, com base nas recomendações de seus membros e dos consultores ad hoc designados para aprimorar o processo avaliativo.

Em relação à disponibilidade dos conteúdos, a Paidéia adota a Licença Creative Commons, CC BY-NC Atribuição não comercial. Com essa licença é permitido acessar, baixar (download), copiar, imprimir, compartilhar, reutilizar e distribuir os artigos, desde que para uso não comercial e com a citação da fonte, conferindo os devidos créditos autorais à revista. Nesses casos, nenhuma permissão é necessária por parte dos autores ou dos editores.

Em decorrência do trabalho exaustivo da Comissão Editorial, os fascículos antigos da revista encontram-se disponibilizados no site da SciELO, a partir da conversão dos arquivos para o padrão utilizado por esta base de dados, ampliando assim a visibilidade dos artigos publicados, em conformidade com a política de acesso livre e universal aos conteúdos.

Outro aspecto que diferencia a Paidéia no cenário das publicações da área de Psicologia é a disponibilização sistemática e periódica de informações sobre o processo de gestão do periódico. No primeiro fascículo de cada ano publicamos um Relatório de Gestão, no qual damos visibilidade ao trabalho consolidado no ano anterior. Esse documento contém indicadores objetivos que permitem aferir o fluxo da revista, como o tempo de tramitação dos manuscritos, a procedência dos autores que têm seus artigos publicados, a origem institucional dos revisores/consultores ad hoc, entre outros tópicos de interesse (Santos, 2010, 2011).

\section{Processo Editorial - 2011}

Ao considerar o processo editorial da Paidéia no ano de 2011, nota-se que o tempo médio de tramitação dos manuscritos foi de, aproximadamente, seis meses. Neste ano, a revista recebeu 99 manuscritos novos, dos quais 15 foram aprovados, 52 recusados, dois tiveram sua tramitação suspensa pelos autores e 30 ainda estavam em processo de avaliação em fevereiro de 2011, quando da redação deste relatório (Tabela 1). Considerando os manuscritos cujo processo editorial foi encerrado em $2011(n=69)$, a revista aprovou $21,7 \%$ e recusou $75,4 \%$. 
Tabela 1

Situação Editorial dos Manuscritos Submetidos à Revista Paidéia em 2011

\begin{tabular}{ll}
\hline \multicolumn{1}{c}{ Parecer final } & $\boldsymbol{n}$ \\
\hline Recebidos (Total) & 99 \\
Aprovados & 15 \\
Recusados & 52 \\
Devolvidos & 2 \\
Em processo editorial & 30 \\
\hline
\end{tabular}

Tempo entre submissão e publicação em $2011=6$ meses

Em 2011, no volume 21 (fascículos 48, 49 e 50), foram publicados 43 artigos, três resenhas, além de três editoriais e um relatório de gestão. As publicações enquadraram-se nas seguintes categorias: (a) relato de pesquisa $(65,2 \%)$; (b) estudo teórico $(19,5 \%)$; (c) resenha $(6,5 \%)$; (d) comunicação breve $(4,3 \%)$; (e) relato de experiência $(4,3 \%)$, como evidencia a Tabela 2.

Tabela 2

Categoria dos Manuscritos Publicados na Revista Paidéia em 2011

\begin{tabular}{lcc}
\hline \multicolumn{1}{c}{ Tipo de manuscrito } & $n$ & $\%$ \\
\hline Relato de pesquisa & 30 & 65,2 \\
Estudo teórico & 9 & 19,5 \\
Resenha & 3 & 6,5 \\
Comunicação breve & 2 & 4,3 \\
Relato de experiência & 2 & 4,3 \\
Total & 46 & 100 \\
\hline
\end{tabular}

A Figura 1 representa a evolução da quantidade de manuscritos submetidos e seu destino no triênio 2009-2011. Em 2011 houve ligeiro aumento do número de manuscritos que se encontravam em processo editorial e diminuição do número de artigos aprovados.

No que concerne à filiação institucional dos autores, a Tabela 3 mostra que a Paidéia publicou artigos provenientes de 25 universidades/faculdades/institutos brasileiros, com discreto predomínio da Universidade Federal de Santa Catarina. Nota-se que os autores dos artigos publicados estão vinculados a uma multiplicidade de instituições de nível superior, de caráter público, privado e confessional, de diversos estados da federação brasileira. Houve predomínio de universidades federais em relação às estaduais.

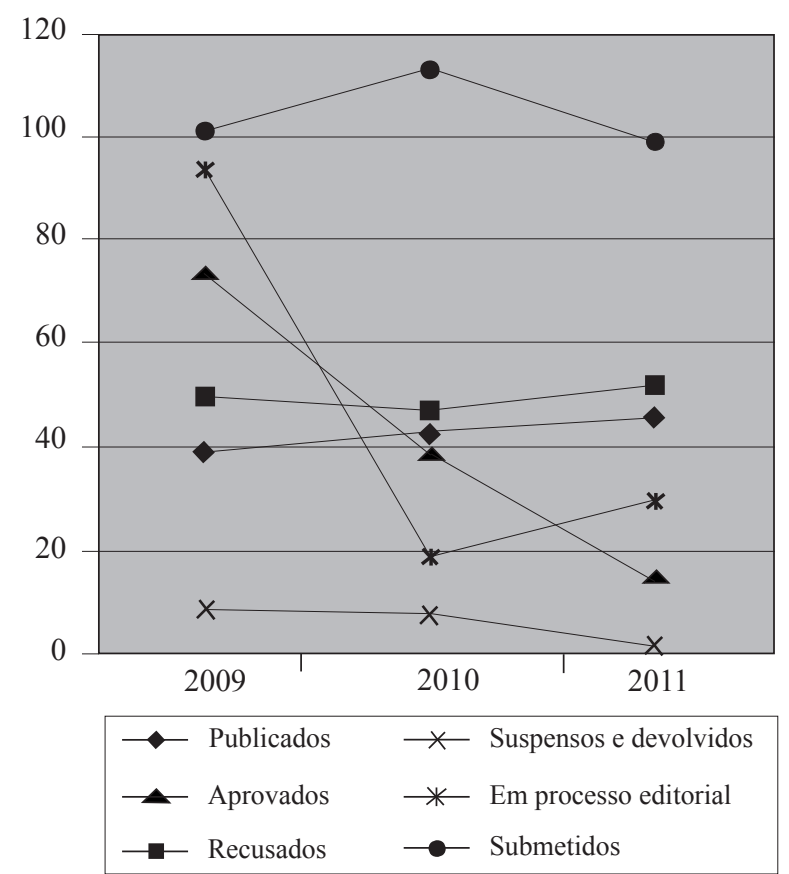

Figura 1. Número de manuscritos submetidos à revista Paidéia e seu desfecho editorial no triênio 2009-2011

Tabela 3

Origem Institucional dos Autores cujos Artigos Foram Publicados na Revista Paidéia em 2011

\begin{tabular}{lll}
\hline \multicolumn{1}{c}{ Instituições nacionais } & $n$ & $\%$ \\
\hline Universidade Federal de Santa Catarina - SC & 6 & 11,5 \\
Universidade de São Paulo - SP & 5 & 9,6 \\
Universidade Federal de São Carlos - SP & 5 & 9,6 \\
Universidade Federal do Rio Grande do Sul - RS & 5 & 9,6 \\
Universidade Estadual Paulista - SP & 4 & 7,7 \\
Pontifícia Universidade Católica - SP, MG & 3 & 5,8 \\
Universidade Federal do Rio de Janeiro - RJ & 2 & 3,8 \\
Universidade São Francisco - SP & 2 & 3,8 \\
Universidade Federal da Paraíba - PB & 2 & 3,8 \\
Universidade Federal de Sergipe - SE & 2 & 3,8 \\
Universidade Federal de Uberlândia - MG & 2 & 3,8 \\
Centro Universitário Salesiano de São Paulo - SP & 1 & 1,9 \\
Faculdade de Medicina de São José do Rio Preto - SP & 1 & 1,9 \\
Universidade Estadual de Campinas - SP & 1 & 1,9 \\
Centro Universitário de Belo Horizonte - MG & 1 & 1,9 \\
Universidade Federal de Juiz de Fora - MG & 1 & 1,9 \\
Universidade Federal de Minas Gerais - MG & 1 & 1,9 \\
Universidade Católica de Brasília - DF & 1 & 1,9 \\
Universidade de Brasília - DF & 1 & 1,9
\end{tabular}

(continua...) 
Tabela 3 (continuação)

\begin{tabular}{|c|c|c|}
\hline Instituições nacionais & $n$ & $\%$ \\
\hline Universidade Federal do Paraná - PR & 1 & 1,9 \\
\hline Universidade Tuiuti do Paraná - PR & 1 & 1,9 \\
\hline Universidade Católica de Pernambuco - PE & 1 & 1,9 \\
\hline Universidade Federal de Pernambuco - PE & 1 & 1,9 \\
\hline Universidade Federal de Goiás - GO & 1 & 1,9 \\
\hline Universidade Federal do Espírito Santo - ES & 1 & 1,9 \\
\hline Total & 52 & 100 \\
\hline Instituições internacionais & $\mathrm{n}$ & $\%$ \\
\hline Universidade de Lisboa, Portugal & 1 & 100 \\
\hline Total & 1 & 100 \\
\hline
\end{tabular}

No que concerne à procedência dos consultores $a d$ hoc, nota-se o predomínio de revisores lotados em universidades do Estado de São Paulo (57\%) que, somados aos avaliadores oriundos de outras instituições pertencentes à região Sudeste, totalizaram $71,9 \%$ do corpo de revisores. A região Sul comportou $13,1 \%$ dos assessores; as regiões Nordeste e Centro-Oeste perfizeram $8,8 \%$ e $2,6 \%$ cada uma e a região Norte, $0,9 \%$ (Tabela 4).

Tabela 4

Abrangência do Corpo de Consultores ad hoc da Paidéia conforme Origem Geográfica dos Avaliadores $(n=114)$

\begin{tabular}{|c|c|c|}
\hline \multirow{2}{*}{$\begin{array}{c}\text { Internacionais } \\
\text { País }\end{array}$} & \multicolumn{2}{|c|}{ Consultores } \\
\hline & $n$ & $\% *$ \\
\hline Portugal & 3 & 2,6 \\
\hline Nacionais & \multicolumn{2}{|c|}{ Consultores } \\
\hline Estado & $n$ & $\% *$ \\
\hline \multicolumn{3}{|l|}{ Região Sudeste } \\
\hline São Paulo & 65 & 57,0 \\
\hline Rio de Janeiro & 11 & 9,6 \\
\hline Minas Gerais & 6 & 5,3 \\
\hline Subtotal & 82 & 71,9 \\
\hline \multicolumn{3}{|l|}{ Região Sul } \\
\hline Rio Grande do Sul & 9 & 7,9 \\
\hline Santa Catarina & 4 & 3,5 \\
\hline Paraná & 2 & 1,7 \\
\hline Subtotal & 15 & 13,2 \\
\hline \multicolumn{3}{|l|}{ Região Nordeste } \\
\hline Bahia & 4 & 3,5 \\
\hline Pernambuco & 4 & 3,5 \\
\hline Ceará & 1 & 0,9 \\
\hline Paraíba & 1 & 0,9 \\
\hline Subtotal & 10 & 8,8 \\
\hline
\end{tabular}

Tabela 4 (continuação)

\begin{tabular}{llc}
\hline \multicolumn{1}{c}{ Nacionais } & \multicolumn{2}{c}{ Consultores } \\
\cline { 2 - 3 } & $n$ & $\%{ }^{*}$ \\
\hline Região Centro-Oeste & 2 & 1,7 \\
$\quad$ Distrito Federal & 1 & 0,9 \\
Mato Grosso & 3 & 2,6 \\
$\quad$ Subtotal & & \\
Região Norte & 1 & 0,9 \\
Pará & 1 & 0,9 \\
Subtotal & & \\
\hline
\end{tabular}

* Cálculo baseado no total de 114 assessores que colaboraram com a revista em 2011.

A Figura 2 mostra a distribuição percentual dos consultores $a d$ hoc segundo a procedência geográfica.

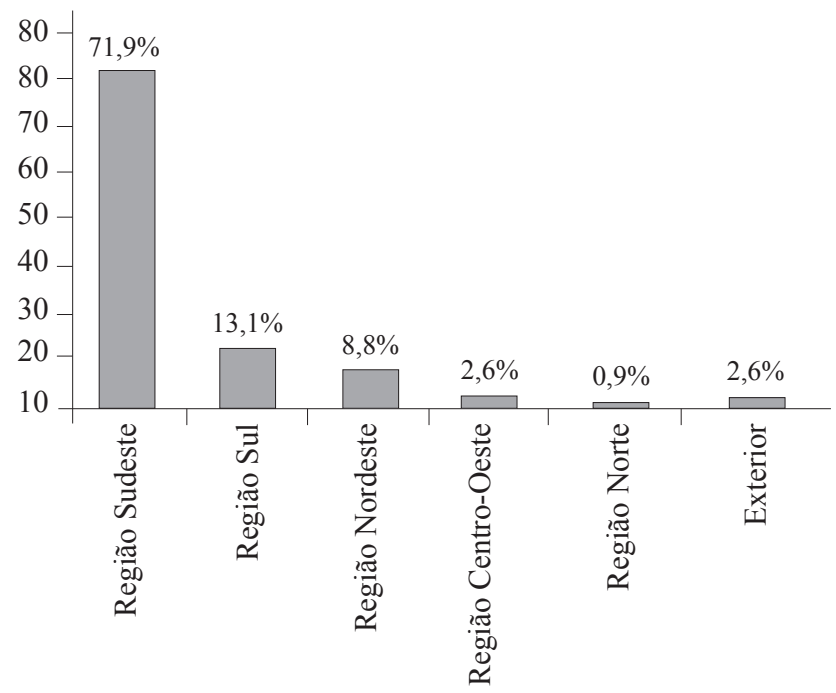

Figura 2. Distribuição dos consultores ad hoc que colaboraram com a Paidéia em 2011 em função da região de origem.

\section{Considerações Finais}

Este Relatório de Gestão sistematiza informações que permitem mapear o quadro da tramitação editorial dos manuscritos submetidos à apreciação da Paidéia durante o ano de 2011. A comparação dos dados acumulados no último triênio mostra que a revista vem fortalecendo seu processo editorial de forma gradual e consistente, consolidando-se como espaço aglutinador do conhecimento produzido no campo da Psicologia e da Educação. No entanto, existem fragilidades que precisam ser consideradas, tais como a morosidade no processo de avaliação dos manuscritos, o baixo percentual de artigos de autores estrangeiros e a excessiva concentração 
de consultores ad hoc em estados da Região Sudeste. A superação desses desafios demanda adoção de estratégias específicas.

Um aspecto positivo a ser destacado no presente relatório é que o índice de "endogenia" da revista (artigos oriundos de autores da Universidade de São Paulo) continua baixo da ordem de 9,6\% dos artigos publicados em 2011. Esse índice era de 9,7\% em 2010 (Santos, 2011) e 18,0\% em 2009 (Santos, 2010).

Como resultado dos esforços continuados que têm sido empreendidos sistematicamente ao longo dos últimos anos, a Paidéia hoje ocupa um lugar destacado entre as publicações da área da Psicologia. No corrente ano foi implantada a submissão online, condição essencial para organizar e gerenciar o fluxo de informações, bem como agilizar o tempo de publicação, que ainda está além do desejável. Os próximos passos consistem em aumentar a visibilidade da revista no âmbito internacional, ampliar a indexação em bases internacionais e aprimorar os indicadores bibliométricos.

Quando se fala em ciência comunicada, é preciso lembrar que a publicação pode se dar por diversos meios, com destaque para os periódicos científicos, devido ao controle de qualidade, maior credibilidade, regularidade e visibilidade da informação. O conhecimento científico é, essencialmente, oriundo de pesquisa original, submetida à avaliação por pares (peer review process) e publicada em revista indexada, com atenção à análise de uso e impacto da informação veiculada. A indexação em bases especializadas ou multidisciplinares é o selo de qualidade de uma revista.

A questão do idioma pátrio, privilegiado na publicação, continua sendo um entrave para o incremento da visibilidade dos artigos editados pelos periódicos nacionais. Há muitos anos a Paidéia já vem publicando resumos em português, inglês e espanhol, o que sempre se constituiu em um diferencial em relação a outras revistas da área da Psicologia, que tradicionalmente só exigem o resumo em português e inglês. A meta agora consiste em implementar a publicação bilíngue dos artigos, ou seja, disponibilizar, na biblioteca eletrônica (SciELO), as versões completas em português e em inglês, seguindo o caminho aberto por revistas de outras áreas do conhecimento. É preciso lembrar que ainda não é tradição, na área da Psicologia, exigir dos autores que os artigos, quando aprovados, sejam publicados concomitantemente em inglês e português, na íntegra. Essa cultura precisa ser rapidamente revista, para que a produção do conhecimento em Psicologia possa alcançar os patamares de excelência desejáveis e se perfilar com outras áreas mais avançadas.

Considerando o aumento considerável da produção científica nacional nos últimos anos, fruto de uma política de estímulo que muitas vezes é confundida com o pernicioso produtivismo resultante da pressão por publicar, é altamente desejável que as revistas nacionais possam dar maior transparência aos seus processos de publicação. Afinal, os periódicos científicos cumprem um papel destacado no processo de disseminação do conhecimento produzido. São os principais meios de publicação e também os de maior prestígio e credibilidade.

\section{Referências}

Comissão Qualis Periódico da Psicologia (2012). Comunicado n ${ }^{\circ}$ 0001/2012 - Área de Psicologia: Atualização do webqualis da área. Brasília: Coordenação de Aperfeiçoamento de Pessoal de Nível Superior. Recuperado em 30 de março 2012, de http://www.capes. gov.br/images/stories/download/avaliacao/Qualis Psicologia.pdf

Santos, M. A. (2010). Paidéia: relatório de gestão - 2009. Paidéia, 20(45), 3-6.

Santos, M. A. (2011). Paidéia: relatório de gestão - 2010. Paidéia, 21(48), 5-8.

Santos, M. A., Risk, E. N., \& Oliveira, V. H. (2009). Paidéia: relatório de gestão - 2008. Paidéia, 19(42), 3-6.

Manoel Antônio dos Santos é Professor Associado da Faculdade de Filosofia, Ciências e Letras de Ribeirão Preto da Universidade de São Paulo, Editor da Paidéia. 